\title{
Prediction of Bitcoin Price Based on the Hidden Markov Model
}

\author{
Muxi $\mathrm{Li}^{1 *}$ \\ ${ }^{1}$ The University of Warwick, Gibbet Hill Rd, Coventry CV4 7AL, UK \\ Corresponding Author's Email: Muxi.Li@warwick.ac.uk
}

\begin{abstract}
The prediction of the financial market has gradually attracted attention from investors. As an emerging market, digital currency has become an indispensable and essential part. The common analysis methods are mainly machine learning and its derivatives. The daily frequency data of Bitcoin prices are obtained in this paper from June 2018 to September 2020. The Hidden Markov Model (HMM) is used to predict Bitcoin prices. In the model, the input observation sequence of the model is selected, including closing price, trading volume, and hidden state. They are in line with the current mainstream market perception: bull market, stable, and bear market. The results show that the relative error of the short-term forecast is relatively low, $0.347 \%$. Compared with traditional models and machine learning methods, the Hidden Markov Model can better identify the state of the digital currency market and predict the direction of price movements, which verifies its application feasibility in the financial market.
\end{abstract}

\section{Keywords: Digital currency, The Hidden Markov Model, Bitcoin price, Short-term forecast}

\section{INTRODUCTION}

Digital currency is usually issued and managed by developers. It is accepted and used by members of a specific virtual community. Cryptocurrencies are a form of internet currency often called digital money or cyber currency. The most important feature of cryptocurrency is that it is not issued by a central bank, nor is it protected by regulations or law, making it impervious to government interference [1]. As a new virtual currency in the financial market, digital currency quickly swept the world due to its low transaction cost, fast transaction speed, and a high degree of anonymity. The fluctuation of the digital currency market reflects the changes in all aspects of society. As the economy continues to develop, digital currencies such as Bitcoin have gradually entered people's lives.

Cash and bank deposits will battle with e-money, electronically stored monetary value denominated in, and pegged to, a common unit of accounts such as the euro, dollar, or renminbi, or a basket thereof. Increasingly popular forms of e-money are stable coins. E-money may be more convenient as a means of payment, but questions arise on the stability of its value [2]. Therefore, accurately predicting the price of digital currency can avoid risks for investors and earn huge assets. As far as the country is concerned, it can make the national economy run smoothly, avoid a series of chain reactions caused by the collapse of the digital currency market. Moreover, it can use technological means to effectively supplement the shortcomings of the existing currency system and serve the social and economic development in a safer, more efficient, and faster way.

Efficient Market Hypothesis (EMH) states that the current market price assimilates all the information available, and the information relevant to a market is contained in the prices [3]. Whenever new information arises, the market corrects itself. Thus, the market is efficient, and there is no space for prediction [4]. Nevertheless, several studies have been performed on the stock data to prove that the market is predictable [5].

The traditional statistical forecasting method is relatively simple to analyze the time series. $\mathrm{Wu}$ et al. verified the three oscillators of the stock market: MACD, RSI, and KDJ to predict the stock market [6], but only for short-term forecasts. It is effective, while unable to adapt to a wider range of circumstances and difficult to adapt to the complex and changeable digital currency market. Due to various factors, the price of digital currency may change at any time, with strong volatility and potential cyclicality. 
With the continuous development of theory, the nonlinear theory is gradually applied to financial time series forecasting. Based on the traditional financial time series model, Wichaidit et al. used the correlation coefficient and the ARIMA model [7] to evaluate the performance of CARIMA based on trend similarity and average error. Compared with the linear regression model, the accuracy is partially improved.

With the help of artificial intelligence methods, Lin et al. used the SVM method to predict the trend of specific stocks and indexes in the Taiwan stock market [8], with an accuracy of $55 \%$ to $65 \%$. Kara et al. used a neural network to predict the Istanbul Stock Exchange National 100 Index [9], with an accuracy of more than $75 \%$. Artificial intelligence methods can obtain better prediction accuracy but are difficult to interpret the model and require a lot of data for training. However, there are not much data in the actual process, and it is difficult to obtain accurate and comprehensive data.

The Hidden Markov Model (HMM) was first proposed by Hamiton et al. [10]. This model is a derivative model of the discrete Markov model and has been improved in subsequent applications. Shi and Weigend applied continuous HMM to the S\&P 500 stock price analysis. They found that the accuracy of the HMM model is higher than that of the GARCH $(1,1)$ model [11]. Thomas, Allen, and Kingsbury used HMM to combine the interest rate process with credit risk and conducted an in-depth study on the term structure [12]. Liew and Siu converted the discrete HMM into a mixed
Gaussian model by linking the parameters and states, which could solve the option pricing problem [13]. HMM is not only accurate in prediction but also interpretable compared to artificial intelligence methods. It uses the principle of data pattern recognition to set random initial parameters for customs clearance and iterate continuously to identify the best data model, which is more intelligent.

This article will introduce Bitcoin data sources and analyze past bull and bear markets in Section 2. Then, Section 3 describes the relevant parameters of HMM and their representative meanings. And appropriate parameters will be set to serve the subsequent fitting. In Section 4, Bitcoin price is predicted. We also explore whether the current model can be improved. Section 5 shows the improvement of this model.

\section{DATA RESEARCH}

Considering the current policy orientation, in order to comprehensively evaluate the prediction performance of HMM, a representative digital currency is selected for analysis and prediction. The data are all from the Yingwei Finance Network (https://cn.investing.com), with a total of 828 samples. We select Bitcoin price data from June 2018 to September 2020. For the daily price data of Bitcoin, the closing price and trading volume are selected. Then, we will estimate the parameters of the model through training data, predict the future closing price of Bitcoin, and evaluate the prediction accuracy of the model through test data, as shown in Figure 1.

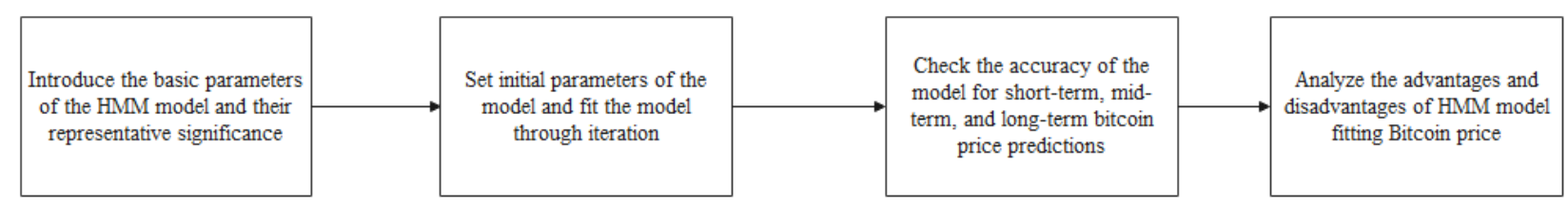

Figure 1 Flow chart 


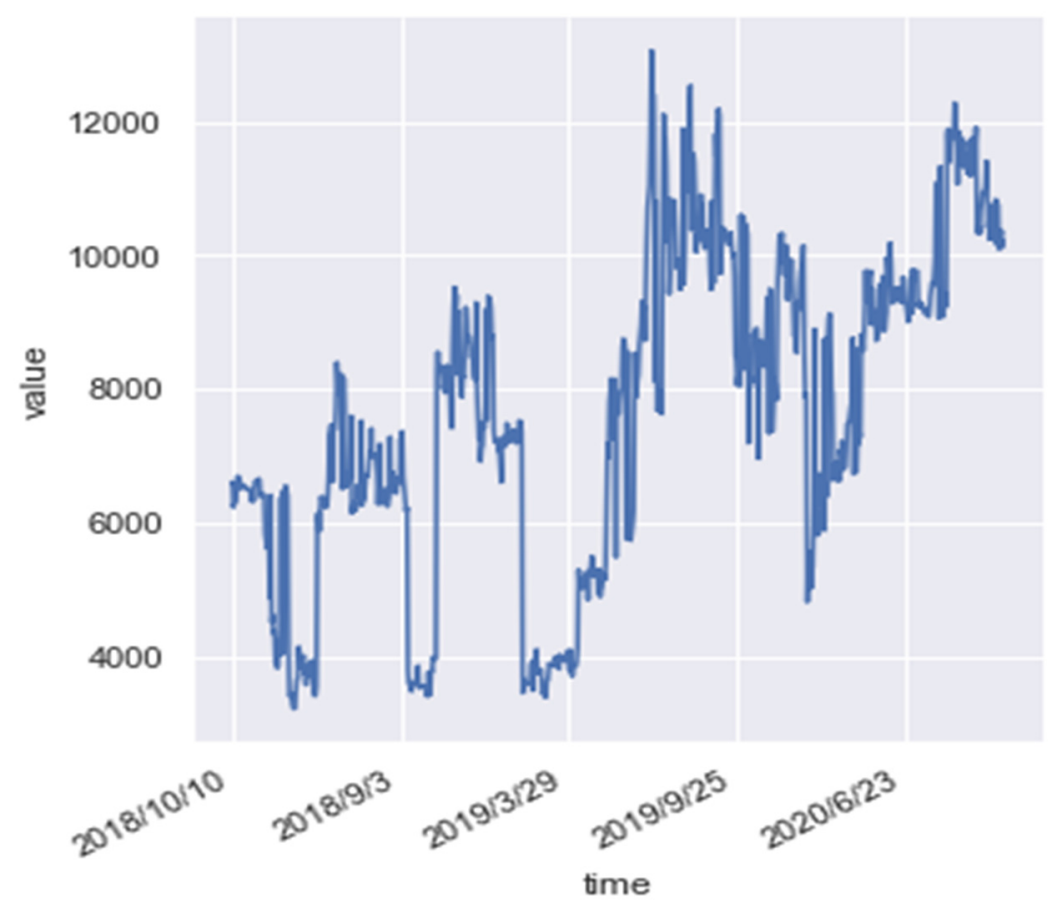

Figure 2 Bitcoin price chart

Figure 2 shows the Bitcoin price (BTC) chart. The Bitcoin bull market started in March 2019, and the sign signal was that the BTC price exceeded $\$ 3,800$ and stood above the 120-day moving average. Although the "312" black swan incident occurred during the period, this rhythm was disrupted. In April 2020, the price of BTC exceeded $\$ 8,000$ and stood above the 120-day moving average, officially declared to enter the bull market. The period lasted for 1 year, an increase of 6 times.

\section{MODELS}

The Hidden Markov model is a probabilistic model about time series. It describes the process of generating a random sequence of unobservable states randomly from a hidden Markov chain and then, generating an observation from each state to generate a random sequence of observations. The key idea is that an HMM is a finite model that describes a probability distribution over an infinite number of possible sequences. [14]

The sequence of states randomly generated by the hidden Markov chain is called the state sequence; each state generates an observation, and the resulting random sequence of observations is called the observation sequence. Each position in the sequence can be regarded as a moment. Let $Q$ be the set of all possible states and $V$ be the set of all possible observations.

$$
Q=\left\{q_{1}, q_{2}, \cdots q_{N}\right\}, V=\left\{v_{1}, v_{2}, \cdots v_{M}\right\}
$$

Among them, $N$ is the number of possible states, and $M$ is the number of possible observations. Suppose $I$ is the state sequence of length $T$, and $O$ is the corresponding observation sequence:

$$
I=\left\{i_{1}, i_{2}, \cdots i_{T}\right\}, O=\left\{o_{1}, o_{2}, \cdots o_{T}\right\}
$$

$A$ is the state transition probability matrix:

$$
A=\left[a_{i j}\right]_{N \times N}
$$

$\pi$ is the initial state probability vector:

$$
\pi=\left(\pi_{i}\right)
$$

The hidden Markov model is determined by the initial state probability vector $\pi$, the state transition probability matrix $A$ and the observation probability matrix $B . \pi$ and $A$ determine the sequence of states, and $B$ determines the sequence of observations. Therefore, the hidden Markov model $\lambda$ can be represented by a ternary symbol, namely

$$
\lambda=(A, B, \pi)
$$

$A, B, \pi$ are called the three elements of the hidden Markov model. The state transition probability matrix $A$ and the initial state probability vector $\pi$ determine the hidden Markov chain and generate an unobservable state sequence. The observation probability matrix $B$ determines how to generate observations from the state, and the state sequence determines how to generate the observation sequence.

The Hidden Markov model has three basic problems: Firstly, the probability calculation problem. Given the model $\lambda=(A, B, \pi)$ and the observation sequence $O=\left\{o_{1}, o_{2}, \cdots o_{T}\right\}$, calculate the probability $P(O \mid \lambda)$. Secondly, the problem of learning. Given the 
observation sequence $O=\left\{o_{1}, o_{2}, \cdots o_{T}\right\}$, estimate the model parameters $\lambda=(A, B, \pi)$ so that the observation sequence probability $P(O \mid \lambda)$ maximum. That is, use the maximum likelihood estimation method to estimate the parameters. Third, the prediction problem, also known as the decoding problem. Given model $\lambda=(A, B, \pi)$ and observation sequence $O=\left\{o_{1}, o_{2}, \cdots o_{T}\right\}$, find the maximum conditional probability $P(I \mid \lambda)$ for a given observation sequence. The state sequence $I=$ $\left\{i_{1}, i_{2}, \cdots i_{T}\right\}$. That is, given the observation sequence, find the most likely corresponding state sequence.

The prediction principle of HMM is pattern recognition. There is no need to obtain a large amount of digital currency price data. In the parameter estimation of the model, an appropriate density function is selected; after data training, the best parameters are found, and finally, the likelihood value is used to find similar data patterns. Model prediction results with high accuracy and can be explained.

HMM uses the likelihood value estimation method to identify a set of data in historical data whose trend is similar to the current trend so as to obtain the predicted value of the digital currency price. First, collect raw data. Look for the digital currency price observation sequence $O=\left\{o_{1}, o_{2}, \cdots o_{T}\right\}$. Then, determine the number of hidden states. Use AIC and BIC criteria to train HMM models with different numbers of states to determine the number of stable hidden states. Next, the model parameters are estimated. The initial parameters are randomly generated, and the Baum-Welch algorithm is used for training and iterates continuously. When the maximum number of iterations is reached, the calculation is stopped, and the estimated model parameters are obtained. Finally, identify similar patterns and obtain predicted values.

HMM model diagram

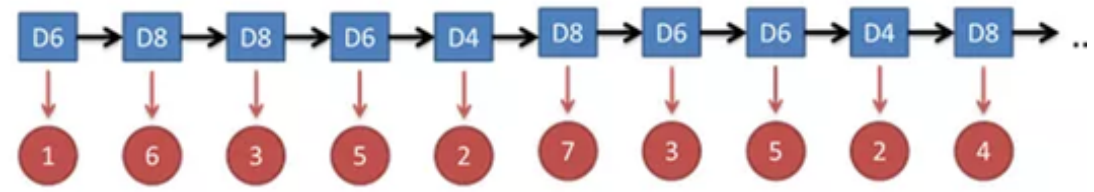

illustration

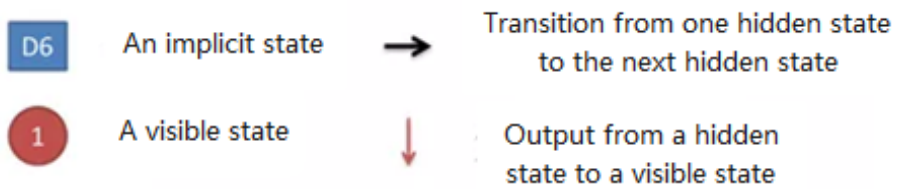

Figure 3 HMM model diagram

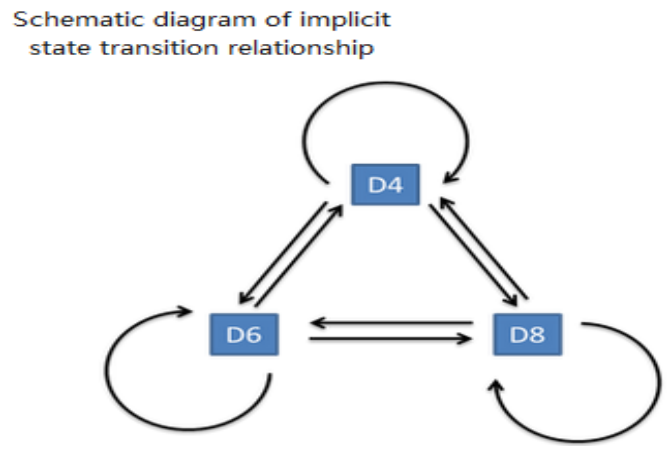

Figure 4 HMM model state transition diagram

\section{RESULTS AND DISCUSSION}

To handle HMM, the classification, optimal-state sequence and training problems should be considered [15]. In the HMM model, there are many parameters. This paper uses traditional Gaussian fitting probability to generate distributions and selects 3 hidden states, which are in line with the current mainstream market perception: bull market, stable, and bear market. In practice, we set the maximum number of iterations to 1000 to avoid underfitting. The covariance matrix is a traditional diagonal matrix.

The HMM model inputs the observation sequence, including closing price and trading volume. We use hmmlearn in python to fit the model and make short-term, mid-term, and long-term predictions for Bitcoin prices. The result obtained is compared with the actual value, as shown in Figure 5. 


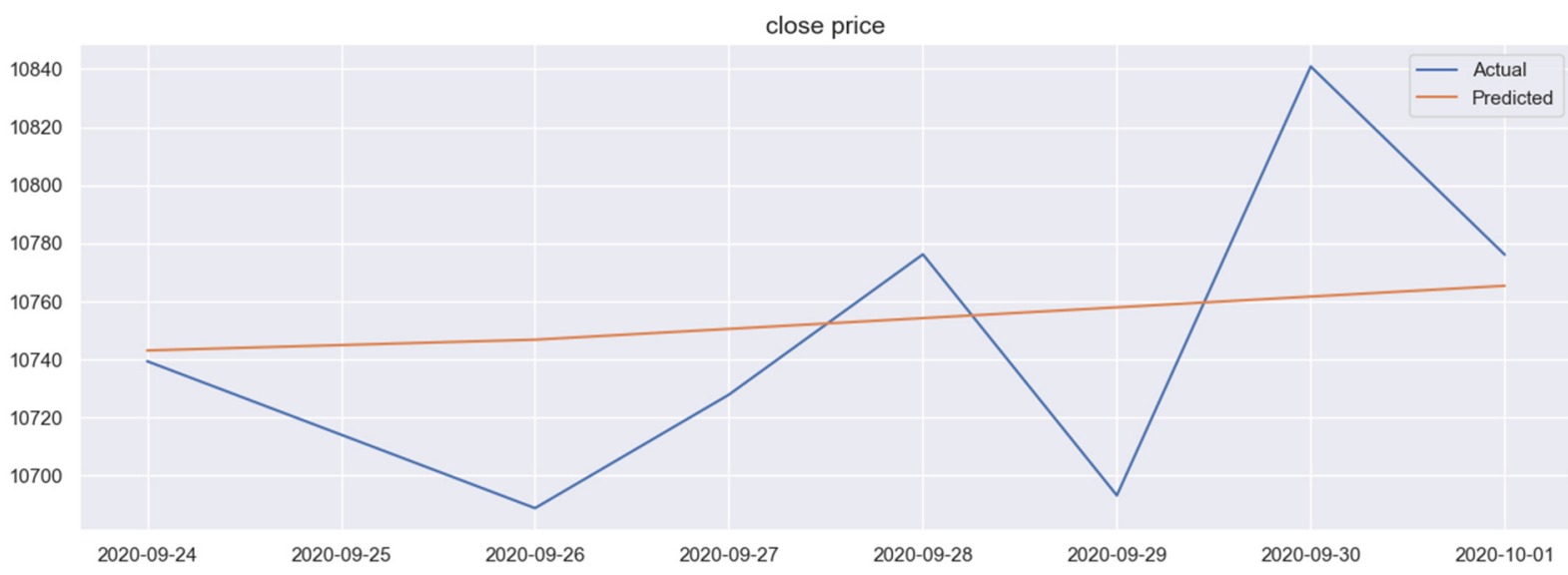

Figure 5. Bitcoin short-term price fitting diagram

It can be seen that the data has great similarities, and based on this, a single-day forecast can be made.

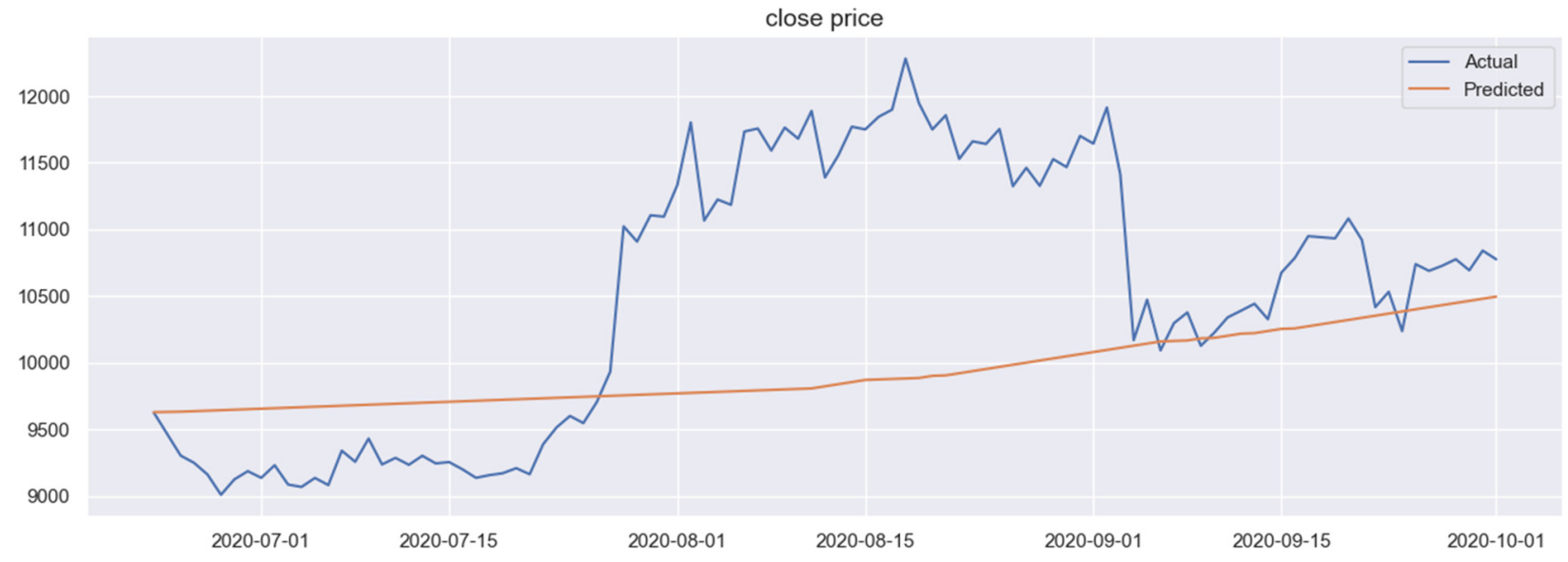

Figure 6. Bitcoin mid-term price fitting chart

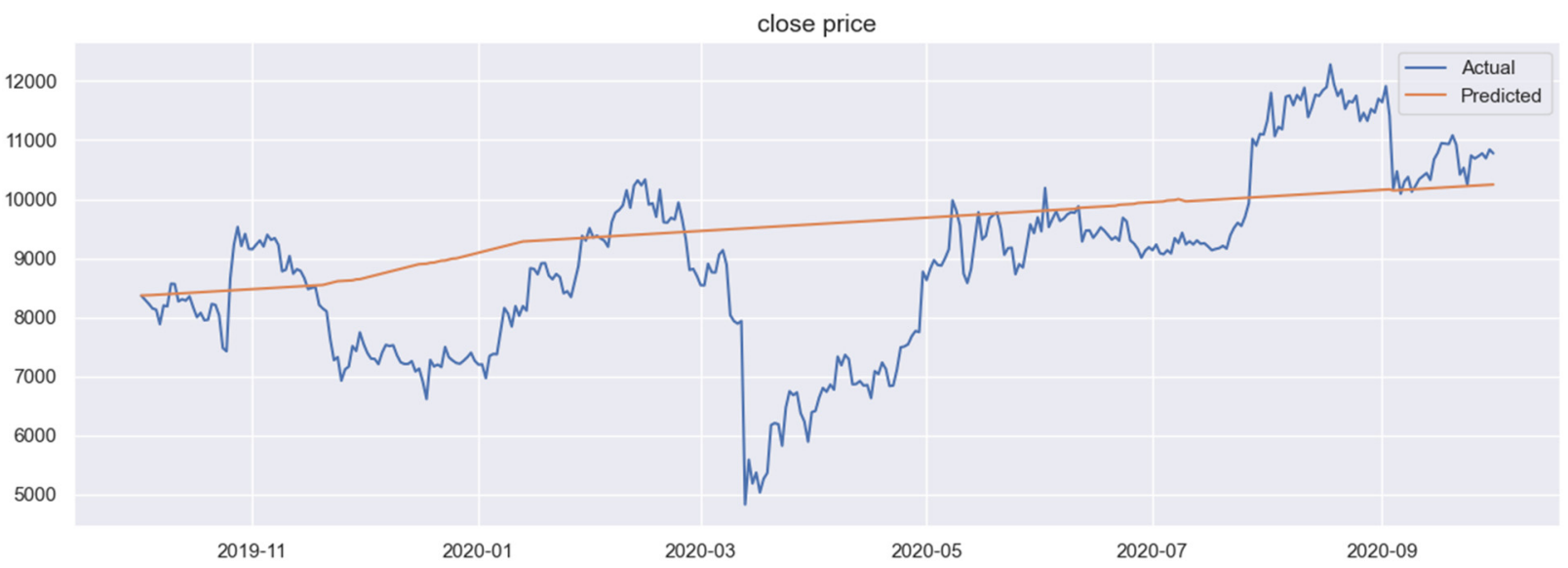

Figure 7. Bitcoin long-term price fitting diagram

Figures 5-7 show the short-term, medium-term and long-term price fitting diagrams of Bitcoin. The results show that when the HMM model is used for short-term prediction, the prediction results and the true value trend are the same, the relative error is small, and the prediction results are better but can't respond well to volatility. When the HMM model is used for medium-term and long-term forecasts, the use of this model will lead to relatively large errors, and it is difficult to effectively predict the digital currency market with large market fluctuations. The predicted error results are shown in Table 1. As time increases, the accuracy of predictions decreases significantly. 
Table 1. Relative error statistics of HMM model

\begin{tabular}{|l|l|l|l|}
\hline & Short-term & Mid-term & Long-term \\
\hline Time span & 7 & 100 & 365 \\
\hline Relative error & $0.346 \%$ & $7.809 \%$ & $13.942 \%$ \\
\hline
\end{tabular}

\section{CONCLUSIONS}

This article uses the Bitcoin closing price data from June 2018 to September 2020 and uses python to fit the HMM model to test the accuracy of the HMM model in the digital currency field. From the experimental results, the HMM model has relatively small short-term prediction errors and can predict the price of Bitcoin very well. At the same time, it is interpretable compared with machine learning technology, so it can be used as an optional model to predict similar digital currencies. However, when using this model to make mid- and long-term forecasts, it is limited by the model's independence assumptions, making it difficult to effectively predict the digital currency market with large market volatility. It is more suitable for forecasting strong growth or long-term decline. This article does not consider noise reduction methods, and at the same time, there is no good way to avoid the possibility of overfitting in the parameter design. Follow-up can improve the model by improving the input parameters and processing the original data to delete the outlier.

\section{REFERENCES}

[1] Árnason S L. Cryptocurrency and Bitcoin. A possible foundation of future currency: why it has value, what is its history and its future outlook[D]. 2015.

[2] Adrian T, Mancini-Griffoli T. The rise of digital money[J]. Annual Review of Financial Economics, 2019, 13 .

[3] Hellström T, Holmström K. Predicting the Stock Market, Technical Report Series[J]. 1998.

[4] Fama E F. Efficient capital markets a review of theory and empirical work[J]. The Fama Portfolio, 2021: 76-121.

[5] Lawrence R. Using neural networks to forecast stock market prices[J]. University of Manitoba, 1997, 333: 2006-2013.
[6] Wu M, Diao X. Technical analysis of three stock oscillators testing MACD, RSI and KDJ rules in SH \& SZ stock markets[C]//2015 4th International Conference on Computer Science and Network Technology (ICCSNT). IEEE, 2015, 1: 320-323.

[7] Wichaidit S, Kittitornkun S. Predicting SET50 stock prices using CARIMA (cross correlation ARIMA)[C]//2015 International Computer Science and Engineering Conference (ICSEC). IEEE, 2015: $1-4$.

[8] Lin Y, Guo H, Hu J. An SVM-based approach for stock market trend prediction[C]//The 2013 international joint conference on neural networks (IJCNN). IEEE, 2013: 1-7.

[9] Kara Y, Boyacioglu M A, Baykan Ö K. Predicting direction of stock price index movement using artificial neural networks and support vector machines: The sample of the Istanbul Stock Exchange[J]. Expert systems with Applications, 2011, 38(5): 5311-5319.

[10] Hamilton J D. A new approach to the economic analysis of nonstationary time series and the business cycle $[\mathrm{J}]$. Econometrica: Journal of the econometric society, 1989: 357-384.

[11] Weigend A S. Time series prediction: forecasting the future and understanding the past[M]. Routledge, 2018.

[12] Thomas L C, Allen D E, Morkel-Kingsbury N. A hidden Markov chain model for the term structure of bond credit risk spreads[J]. International Review of Financial Analysis, 2002, 11(3): 311-329.

[13] Liew C C, Siu T K. Martingale representation and admissible portfolio process with regime switching[J]. Stochastic Analysis and Applications, 2010, 29(1): 106-120.

[14] Eddy S R. Hidden markov models[J]. Current opinion in structural biology, 1996, 6(3): 361-365.

[15] Mohamed M A, Gader P. Generalized hidden Markov models. I. Theoretical frameworks[J]. IEEE Transactions on fuzzy systems, 2000, 8(1): 67-81. 